\title{
ESTIMATING THE CODE BIAS OF BDS TO IMPROVE THE PERFORMANCE OF MULTI- GNSS PRECISE POINT POSITIONING
}

\author{
Jian CHEN ${ }^{1)}{ }^{*}$, Dongjie YUE ${ }^{1)}$, Zhiqiang LIU ${ }^{1)}$, Shaolin $\mathrm{ZHU}^{1)}$ and Xingwang ZHAO ${ }^{2)}$ \\ ${ }^{1)}$ School of Earth Science and Engineering, Hohai University, Nanjing, 211100, China \\ ${ }^{2)}$ School of Geodesy and Geomatics, Anhui University of Science and Technology, Huainan, 232001, China
}

*Corresponding author's e-mail: CJ_19930815@163.com

\begin{tabular}{l} 
ARTICLE INFO \\
\hline Article history: \\
Received 8 September 2018 \\
Accepted 11 December 2018 \\
Available online 13 December 2018 \\
\hline
\end{tabular}

\section{Keywords:}

Code bias

Empirical mode decomposition

Multiple global navigation satellite

systems precise point positioning

Positioning accuracy

Convergence time

\begin{abstract}
An empirical mode decomposition (EMD) model for BeiDou Navigation Satellite System (BDS) code bias has been established upon the observation model of multiple global navigation satellite systems (multi-GNSS). To validate the correctness and effectiveness of the model, seven days from day of year (DOY) 213-219, 2015 from eight Multi-GNSS Experiment (MGEX) stations were processed. Results show that after code bias correction, the standard deviation of the multipath combination (MP) series on B1 and B2 frequencies decreased by $38.63 \%$ and $17.4 \%$, respectively. The timespan needed for convergence in BDS precise point positioning (PPP) was improved by $7.9 \%$ after inclined geosynchronous orbit (IGSO) and medium earth orbit (MEO) code bias correction, and another improvement of $11.4 \%$ was generated by applying geostationary orbit (GEO) code bias correction. Despite the improvement of convergence time, the accuracy of the single-day solution barely increased for PPP in multi-GNSS as compared to the single GNSS. A continuous decrease in percentage along with prolonged timespan for PPP convergence was observed with increasing cut-off elevation angle. However, the performance of multi-GNSS PPP, which was superior to that of the single GNSS, shows that it is extremely valuable for practical applications in mountainous or sheltered areas.
\end{abstract}

\section{INTRODUCTION}

The advent of the BeiDou Navigation Satellite System (BDS) and Galileo Satellite Navigation System (Galileo), as well as the restoration and improvement of Global Navigation Satellite System (GLONASS), has promoted the single global positioning system (GPS) to evolve gradually into multiple global navigation satellite systems (multiGNSS). Advantages for such systems include an enhanced accuracy of positioning plus higher reliability and better availability, which are especially significant for challenging environments such as urban areas and ravines (Li et al., 2015; Cai et al., 2015; Guo et al., 2016; Abd Rabbou et al., 2016). Actually, multi-GNSS precise point positioning (PPP) has already shown its potential as a leader in GNSS precise positioning in the future.

Initially, research on combination PPP was primarily founded upon the combined GPS/GLONASS systems. Functional and stochastic models of PPP that use GPS/GLONASS have been deduced from the model of ionosphere-free observation. Experiments suggest that an improved speed of convergence accompanied with unchanged accuracy of positioning is obtained for PPP in combined systems relative to that in single GPS (Cai et al., 2012). When fewer GPS satellites are used, integrating GPS with GLONASS is conducive to better PPP performance on accuracy of positioning and timespan needed for convergence (Martin et al., 2011). At the same time, higher accuracy for the initial ambiguity solution is achieved in this combined system, followed by less ambiguity fixed duration (Jokinen et al., 2013; Li et al., 2013). On December 27, 2012 the Chinese BeiDou system started to serve the Asia-Pacific region for timing, navigation, and positioning. Studies point out that PPP in GPS/BDS is slightly inferior to the GPS-only system, probably due to the multipaths of BDS geostationary orbit (GEO) satellites (Zhao et al., 2013). Compared with GPSonly or GLONASS-only, improved convergence time was obtained for PPP in combined GPS/GLONASS/BDS PPP, but the improvement of positioning accuracy was quite limited, as suggested by the 24-hour data processing (Zhao et al., 2016). Which much research has been done, shortcomings still exist. (1) Previous studies have mainly concentrated on combined models, such as the dualsystem GPS/GLONASS and GPS/BDS and the threesystem GPS/GLONASS/BDS, so the PPP solution in the GPS/GLONASS/BDS/Galileo model still needs to be performed. (2) The current BDS PPP model neglects the code bias caused by pseudo-range observations of GEO satellites, whose magnitude reaches the meter-level. This may produce a negative impact on positioning performance. 
The code bias of BDS consists of two categories, the bias of inclined geosynchronous orbit and medium earth orbit (IGSO/MEO) satellites with varied elevations and the bias of GEO satellites. The former category was proposed by Hauschild et al. (2012) and was later found to correlate with satellite elevation and frequency (Montenbruck et al., 2012). In 2014, correction models for IGSO and MEO satellites were built upon the correlation characteristics between elevation and code bias so as to mitigate the bias's influence (Wanninger et al., 2014). An algorithm of code noise and multipath correction (CNMC) was proposed by Wu et al. (2012) for the second category in order to weaken the code bias of GEO and IGSO satellites; the code biases improved by $60 \%$ and $42 \%$ in the observation of GEO and IGSO satellites, respectively. Later, long-term characteristics of the multipath error time series were analyzed for GEO satellites via filtering out the errors of low frequency; positioning accuracy was thus effectively improved (Wang et al., 2015). An elevation correction model of the GEO satellites has recently been established with a constant correction value, because the elevation of GEO satellite is almost invariable (Lou et al., 2017). PPP with higher accuracy was realized, but certain drawbacks still await to be overcome. For instance, limited modeling data are insufficient to support a high-precision model; in addition, characteristic studies on this code bias GEO satellite have not been fully developed.

In this contribution, the observation model and data processing strategy of PPP in the four-system GPS/GLONASS/BDS/Galileo are expounded at first in Section 2. Afterwards, a methodology of correcting BDS GEO code bias has been proposed on the basis of empirical mode decomposition (EMD) in Section 3.The static multi-GNSS PPP solution is mainly evaluated via data from eight MGEX reference stations in Section 4, with respect to the accuracy of positioning and timespan needed for convergence. Finally, important conclusions accompanied by experimental results are summarized briefly in Section 5.

\section{MODEL OF MULTI-GNSS PPP OBSERVATION AND STRATEGY FOR DATA PROCESSING \\ 2.1. MULTI-GNSS PPP OBSERVATION MODEL}

In PPP, observation equations including $P$ of pseudo-range and $\Phi$ of the carrier phase shown below are utilized to ensure effective data from observation.

$\left\{\begin{array}{l}P_{i}^{s}=\rho_{r}^{s}+c \cdot \delta t_{r}+c \cdot \delta t_{\mathrm{sys}}^{j}-c \cdot \delta t^{s}+M_{\mathrm{d}}^{s} \cdot \delta_{\mathrm{zhd}}+M_{\mathrm{w}}^{s} \cdot \delta_{\mathrm{zwd}}+I_{i}^{s}+c \cdot\left(b_{r, i}-b_{i}^{s}\right)+\varepsilon_{p} \\ \Phi_{i}^{s}=\rho_{r}^{s}+c \cdot \delta t_{r}+c \cdot \delta t_{\mathrm{sys}}^{j}-c \cdot \delta t^{s}+M_{\mathrm{d}}^{s} \cdot \delta_{\mathrm{zhd}}+M_{\mathrm{w}}^{s} \cdot \delta_{\mathrm{zwd}}-I_{i}^{s}+\lambda_{i}\left(N_{i}^{s}+B_{r, i}+B_{i}^{s}\right)+\varepsilon_{\Phi}\end{array}\right.$

where $s, i$, and $r$ denote different satellites, carrier frequencies, and receivers, respectively; $j$ represents the navigation system, as GPS, GLONASS, BDS, Galileo, respectively; $\rho_{r}^{s}$ represents the geometric distance while $c$ is the fixed symbol for light speed; $\delta t_{r}$ and $\delta t^{s}$ refer to clock errors of the receiver and satellite, respectively; $\delta t_{\text {sys }}^{j}$ is the inter-system bias (ISB) (for GPS, the $\delta t_{\text {sys }}^{j}$ is 0 ); $M_{\mathrm{d}}^{s}$ and $M_{\mathrm{w}}^{s}$ are the dry and wet tropospheric mapping function, respectively; $\delta_{\mathrm{zhd}}$ and $\delta_{\mathrm{zwd}}$ are the dry and wet zenith tropospheric delay, respectively; $b_{r, i}$ and $b_{i}^{s}$ are the pseudo-range hardware delay for receiver and satellite, respectively; $B_{r, i}$ and $B_{i}^{s}$ are the carrier phase hardware delay for receiver and satellite, respectively; $I_{i}^{s}$ represents the ionospheric delay of frequency $i ; N_{i}^{s}$ is recorded for the parameter of integer ambiguity while $\lambda_{i}$ represents the wavelength at various frequencies; $\varepsilon_{\mathrm{p}}$ and $\varepsilon_{\Phi}$ are the total of measurement noises and observation multipath errors, respectively.

The hardware delay in the receiver end changes slowly in a short time, so it can be regarded as a constant (Ge et al., 2007; Liu et al., 2015; Liu et al., 2017). For the delay of the different frequencies in the receiver end, the following form can be expressed:

$$
\left\{\begin{array}{l}
b_{r, i}=b_{r}^{a v g}+\delta b_{r, i} \\
B_{r, i}=B_{r}^{a v g}+\delta B_{r, i}
\end{array}\right.
$$

where $b_{r}^{a v g}$ and $B_{r}^{a v g}$ are the mean hardware delays for the pseudo-range and the carrier phase in the receiver end, respectively; $\delta b_{r, i}$ and $\delta B_{r, i}$ are the frequency-dependent delays, respectively.

In Eq. (2), the mean pseudo-range hardware delay bias is assimilated into the receiver clock offset. The pseudorange hardware delay bias for the satellite is contained in the precise clock product provided by the International GPS Service (IGS) analysis centers and it can be removed at the user site when applying the products. The carrier phase hardware delay biases for the satellite can be absorbed by the ambiguities if the ambiguities are not fixed. For the inter-system bias receiver is dependent, thus it can be grouped into the receiver clock. Above all, the clock errors of the receiver and satellite and integer ambiguity are redefined as:

$$
\left\{\begin{array}{l}
\delta \bar{t}_{r}^{j}=\delta t_{r}+b_{r}^{a v g}+\delta t_{\mathrm{sys}}^{j} \\
\delta \bar{t}^{s}=\delta t^{s}+b_{i}^{s} \\
\bar{N}_{i}^{s}=N_{i}^{s}+B_{r, i}+B_{i}^{s}-c \cdot\left(b_{r}^{a v g}-b_{i}^{s}\right) / \lambda_{i}
\end{array}\right.
$$


Thus, applying Eq. (3) in Eq. (1), Eq. (1) can be rewritten as follows:

$$
\left\{\begin{array}{l}
P_{i}^{s}=\rho_{r}^{s}+c \cdot \delta t_{r}^{j}-c \cdot \delta t^{s}+M_{\mathrm{d}}^{s} \cdot \delta_{\mathrm{zpd}}+M_{\mathrm{w}}^{s} \cdot \delta_{\mathrm{zpw}}+I_{i}^{s}+\varepsilon_{p} \\
\Phi_{i}^{s}=\rho_{r}^{s}+c \cdot \delta t_{r}+c \cdot \delta t_{\mathrm{sys}}^{j}-c \cdot \delta t^{s}+M_{\mathrm{d}}^{s} \cdot \delta_{\mathrm{zhd}}+M_{\mathrm{w}}^{s} \cdot \delta_{\mathrm{zwd}}-I_{i}^{s}+\lambda_{i}\left(N_{i}^{s}+B_{r, i}+B_{i}^{s}\right)+\varepsilon_{\Phi}
\end{array}\right.
$$

In order to analysis the performance of the combined PPP, Eq. (5) gives the University of Calgary (UofC) model. The model not only eliminates the delay of the one-order ionosphere, it also has smaller pseudo-range observation noise. The function model expressed as follows:

$$
\left\{\begin{aligned}
L_{P \Phi} & =\frac{P_{i}+\Phi_{i}}{2} \\
& =\rho_{r}^{s}+c \cdot \delta \bar{t}_{r}^{j}-c \cdot \delta \bar{t}^{s}+M_{\mathrm{d}}^{s} \cdot \delta_{\mathrm{zpd}}+M_{\mathrm{w}}^{s} \cdot \delta_{\mathrm{zpw}}+\frac{\lambda_{i} \bar{N}_{i}^{s}}{2}+\varepsilon_{L_{P \Phi}} \\
\Phi_{\mathrm{IF}} & =\frac{f_{1}^{2} \cdot \Phi_{1}-f_{2}^{2} \cdot \Phi_{2}}{f_{1}^{2}-f_{2}^{2}} \\
& =\rho_{r}^{s}+c \cdot \delta t_{r}^{j}-c \cdot \delta \bar{t}^{s}+M_{\mathrm{d}}^{s} \cdot \delta_{\mathrm{zpd}}+M_{\mathrm{w}}^{s} \cdot \delta_{\mathrm{zpw}}+\lambda_{\mathrm{IF}} \bar{N}_{\mathrm{IF}}^{s}+\varepsilon_{\Phi_{\mathrm{IF}}}
\end{aligned}\right.
$$

where $\lambda_{\mathrm{IF}}$ is the wavelength of ionosphere-free observations, and $N_{\mathrm{IF}}$ is the integer ambiguity of the ionospherefree.

\subsection{DATA PROCESSING STRATEGY}

In this contribution, the UofC model with an extended Kalman filter is applied for parameter estimation, and parameters being estimated include the receiver position, wet tropospheric delay, receiver clock error, and ambiguities. The phase center offset (PCO) and phase center variation (PCV) for GPS and GLONASS can refer to the ANTEX file released by the IGS (Ren et al., 2015).The ANTenna Exchange format (ANTEX) file can also be referenced for the PCO at satellite ends in BDS and Galileo, whereas no organization provides the corresponding PCV at satellite ends or both PCO/PCV at the receiver (Zhang et al., 2015). The observation, error correction, and parameter estimation are summarized in Table 1:

\section{BDS CODE BIAS ANALYSIS}

To validate the GEO code bias model and the multi-GNSS PPP performance, the observation data are gathered from eight Multi-GNSS Experiment (MGEX) reference stations distributed in the Asia Pacific region (Fig. 1) from August 1, 2015 till August 7, 2015 with a sampling rate of $30 \mathrm{~s}$. Reference stations are environmentally ideal for observation and less affected by multipath.

Multipath error always exists in BDS measurements. A mathematical model of the pseudo-range multipath combination can be found through combining the pseudo-range and carrier phase observations as shown below:

$\mathrm{MP}_{i}=P_{i}-m_{i j} \cdot \lambda_{i} \varphi_{i}+\left(m_{i j}-1\right) \cdot \lambda_{j} \varphi_{j}+B_{i}$

\begin{tabular}{|c|c|c|}
\hline & Parameters & Models \\
\hline \multirow{5}{*}{ Observation } & Observations & Four-system pseudo-range and carrier phase observation \\
\hline & Signal & GPS: L1, L2; GLONASS: L1, L2; BDS: B1, B2; Galileo: E1, E5a \\
\hline & Sampling rate & $30 \mathrm{~s}$ \\
\hline & Elevation cutoff & $7^{\circ}$ \\
\hline & Observation weight & Elevation-dependent weight \\
\hline \multirow{8}{*}{$\begin{array}{l}\text { Error } \\
\text { correction }\end{array}$} & Phase-windup effect & Corrected \\
\hline & $\begin{array}{l}\text { Receiver antenna phase } \\
\text { center }\end{array}$ & $\begin{array}{l}\text { PCO and PCV values for GPS and GLONASS from igs08.atx are used; } \\
\text { PCO and PCV values for BDS and Galileo are not considered }\end{array}$ \\
\hline & Satellite antenna phase center & PCO and PCV values for GPS and GLONASS from igs08.atx are used; \\
\hline & & $\begin{array}{c}\text { PCO values for Galileo and BDS from igs08.atx are used, while PCV } \\
\text { values are not considered }\end{array}$ \\
\hline & Relativistic effect & Corrected \\
\hline & Satellite orbit and clock & MGEX (German Research Centre for Geosciences GFZ) products \\
\hline & Ionospheric delay & Ionosphere-free combination \\
\hline & Dry tropospheric delays & Corrected \\
\hline \multirow{4}{*}{$\begin{array}{l}\text { Parameter } \\
\text { estimation }\end{array}$} & Receiver coordinates & Estimated \\
\hline & Receiver clock & Estimated \\
\hline & Phase ambiguities & Estimated \\
\hline & Wet tropospheric delays & Estimated \\
\hline
\end{tabular}

Table 1 Processing strategy of static PPP for the multi-GNSS. 


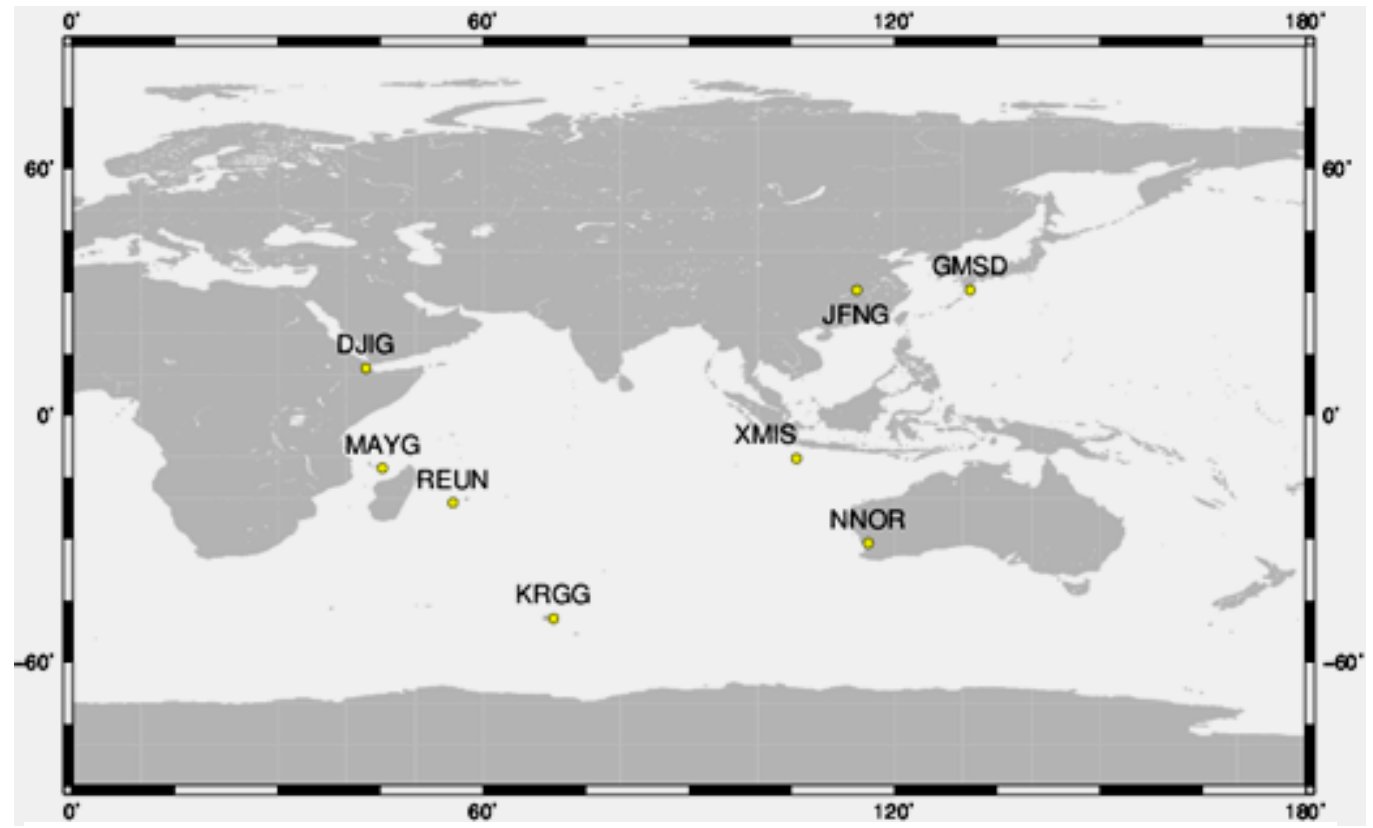

Fig. 1 Distribution of MGEX stations.

$m_{i j}=\frac{f_{i}^{2}+f_{j}^{2}}{f_{i}^{2}-f_{j}^{2}}$

where $f$ is the frequency and $\lambda$ is the wavelength; the subscripts $i, j$ represent different frequencies; $\varphi$ and $P$ represent the carrier phase observation and the pseudo-range observation as mentioned before; phase ambiguity, hardware bias, and multipath error are embodied by $B$.

Clearly, ambiguity and hardware bias combinations, the error of pseudo-range multipath, and random noises are all covered by the above equations, while the geometric satellite-receiver distance, clock error, along with the atmosphere and other receiver-specific delays have been eliminated by Eq. (6). When no cycle slip occurs, $B$ is assumed to be a constant and can be derived through over epochs (Zhu et al., 2015).

Figure 2 presents the BDS multipath (MP) and elevation series under $\mathrm{B} 1$ and $\mathrm{B} 2$ frequencies based on the GMSD station. The observation data from the $\mathrm{C} 07$ and $\mathrm{C} 12$ satellites were collected during $7 \mathrm{~h}$ on August 1, 2015, while those from the C01 satellite are MP series, coming from two consecutive days during August 1 - 2, 2015 (DOY213 - DOY214).

As shown in Figure 2, a systematic error occurs in the MP time series of MEO and IGSO satellites and it decreases with increasing elevation. This is

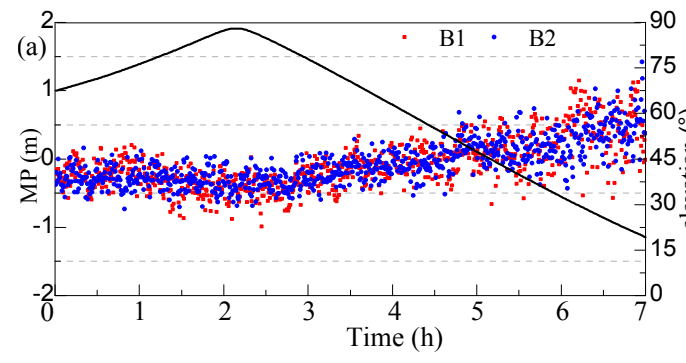

(a) $\mathrm{C} 07$

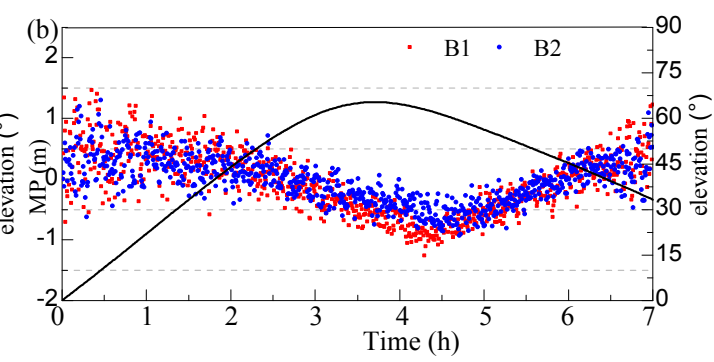

(b) $\mathrm{C} 12$

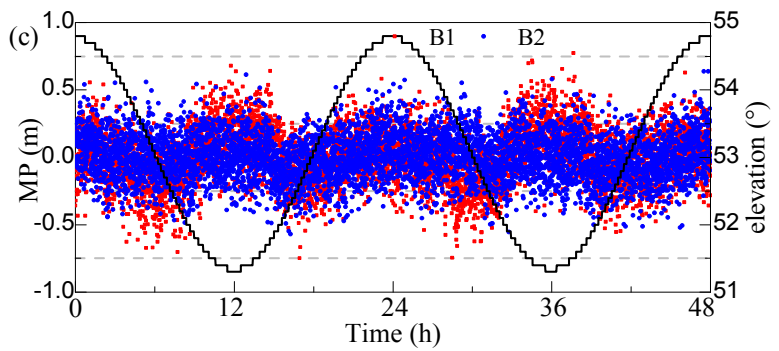

(c) $\mathrm{C} 01$

Fig. 2 BDS MP series and elevation series on B1/B2 frequency. 


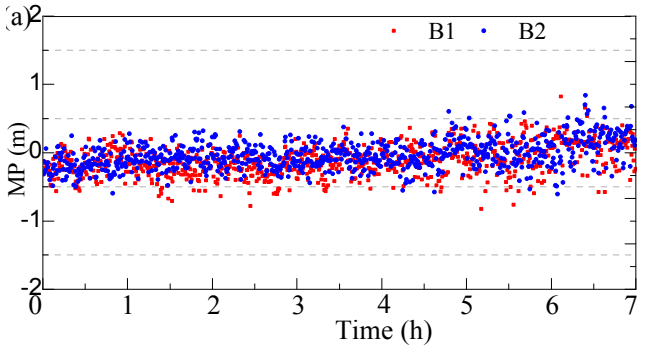

(a) $\mathrm{C} 07$

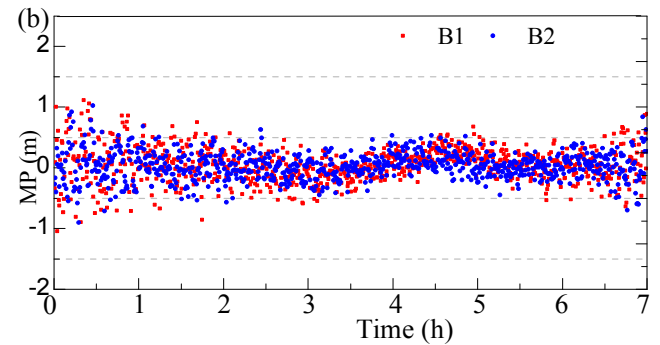

(b) $\mathrm{C} 12$

Fig. 3 MP series after code bias correction.

considered as a code bias, which affects the GEO satellite. The MP time series exhibits fluctuation throughout the day. However, the small elevation range has limited an obvious correlation between code bias and elevation angle. There are apparent differences in the MP time series corresponding to different frequencies.

Code bias is corrected by the Wanninger model for MEO and IGSO satellites, and the corrected MP series are depicted in Figure 3. The code biases of the IGSO and MEO satellite MP series are significantly weakened after model correction.

Since the code bias has regularity of frequency and daily periodicity, a certain mathematical method is used to extract code bias model. The daily periodicity characteristics are used to process the successive days of observation data. The finite impulse response (FIR) filter and wavelet transform

are used to extract code bias model, and both of them obtained good results. However, the FIR filter is affected by the Fourier transform, time-frequency analysis cannot be performed at the same time, and the wavelet filter is restricted by the selection of the wavelet basis. In this research, self-adaptability of EMD has been fully utilized for EMD decomposition of the original signal. EMD is used to decompose data from high frequency to low frequency in the time domain. The code bias and random noise are separated, the code bias model is extracted, and the observation data of the next day is corrected. The detailed description is as follows:

1. The original signal is decomposed adaptively by the EMD model to obtain $n$ intrinsic mode functions (IMFs) with different frequencies as well as a single residual term:

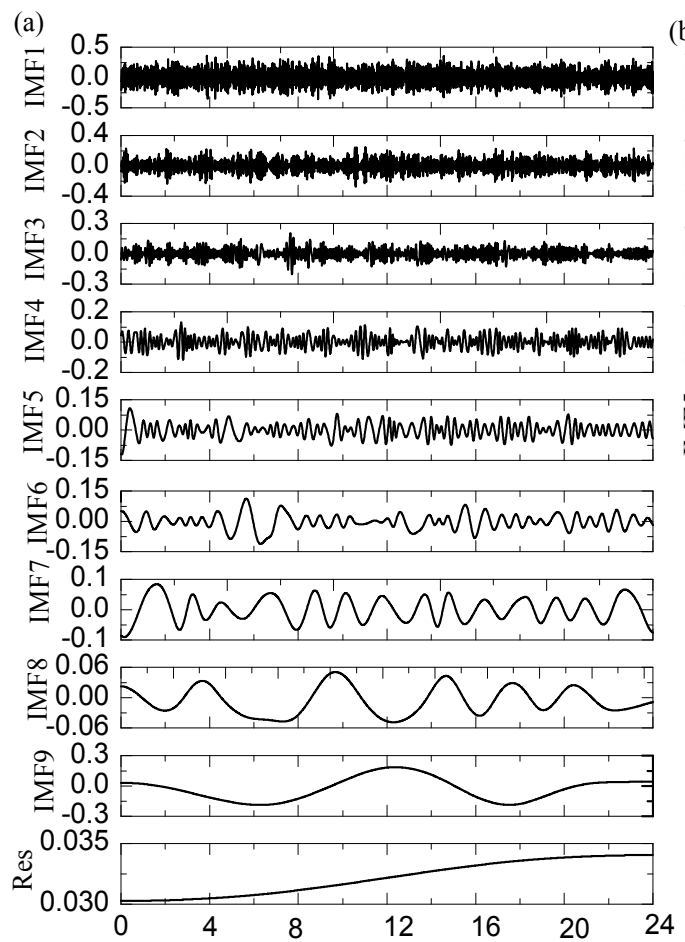

(a) DOY213

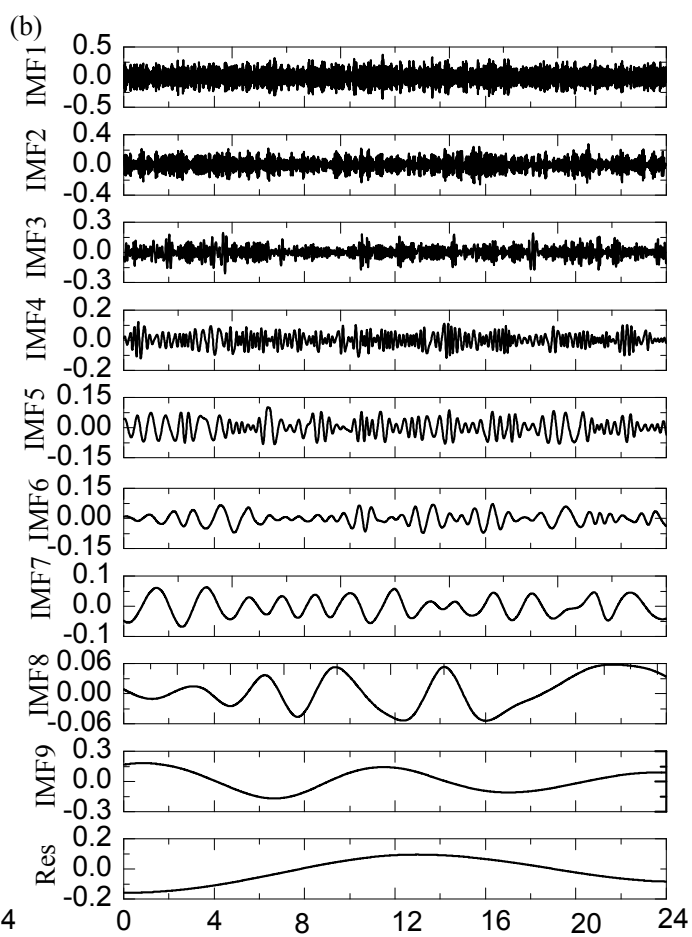

(b) DOY214

Fig. 4 Multi-scale EMD decomposition. 

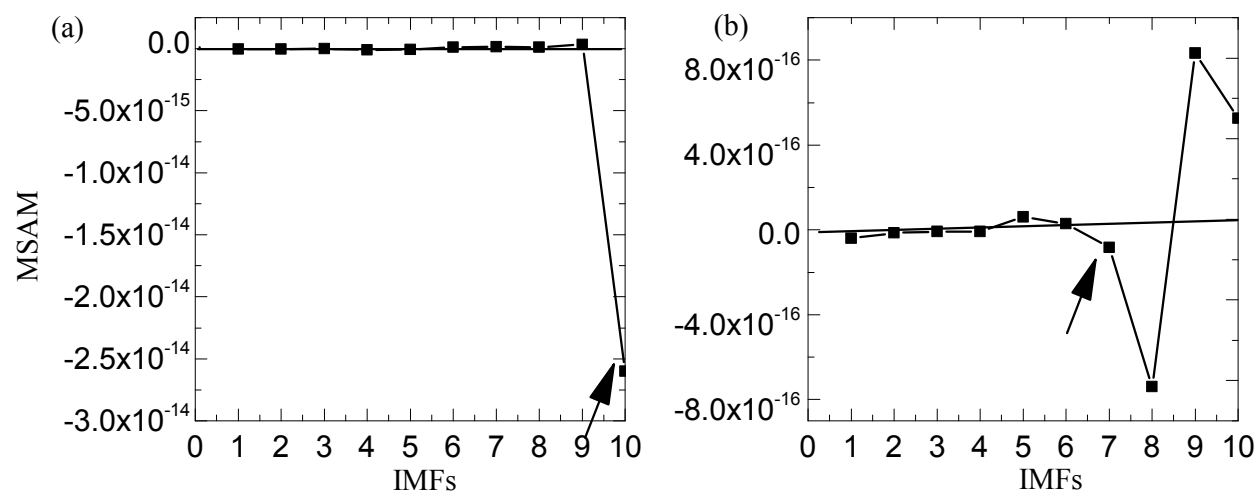

Fig. 5 Relationship between scale and MSAM.

$$
\mathrm{MP}(t)=\sum_{i=1}^{n} i m f_{i}(t)+r_{n}(t)
$$

2. The mean of the standardized accumulated modes (MSAM) for different decomposition layers in the EMD method is calculated with the following equation:

MSAM $=$

$$
=\text { mean }\left(\sum_{i=1}^{m}(\operatorname{IMF}(i)-\operatorname{mean}(\operatorname{IMF}(i)) / \operatorname{std}(\operatorname{IMF}(i)))\right), m \leq n
$$

In Eq. (9), mean function is abbreviated as mean $(\bullet)$ while standard deviation function $\operatorname{as} \operatorname{std}(\bullet)$.

3. MSAM obtained from step (2) is judged in the following manner: an $m^{\text {th }}$ layer is considered to generate a systematic deviation if it shows a significantly deviation of the MSAM value from the zero value. Meanwhile, $1-(m-1)$ IMF is a high-frequency noise.

The MP series for $\mathrm{C} 01$ at the GMSD station during August 1-2, 2015 was processed using EMD model. According to Figure 4, the MP decomposition scale for two consecutive days is 9 , where 9 IMF plus 1 residual term can be obtained. The correlation of MSAM to the scale is demonstrated in Figure 5.
High-frequency and low-frequency IMF can be discriminated when the scale of MP series equals 7 on August 1, 2015. All the IMFs at high frequency (IMF1 - IMF6) have been de-noised, and code bias can be extracted from the EMD reconstruction. The extraction of code bias from the MP series follows the same procedure on August 2, 2015, and the crosscorrelation of code biases on these two consecutive days is greater than 0.98 (Fig. 6). Moreover, it is confirmed that the code bias on certain days can be corrected with the correction value on the previous day. Figure 7 compares the accuracies of uncorrected and corrected MP values for four GEO satellites at the GMSD station. Significant improvement in accuracy was found for the corrected MP series, and frequencies for B1 and B2 were improved by $39.9 \%$ and $29.4 \%$, respectively.

Three schemes are utilized in the BDS static PPP to verify the correction model of code bias: Scheme 1, uncorrected code bias; Scheme 2, corrected code bias for IGSO/MEO satellites only; Scheme 3, corrected code biases for both IGSO/MEO/GEO satellites and GSO/MEO satellites. Positioning error refers to the difference between the positioning solution and the IGS weekly solution, and filtering convergence is defined as the situation when positioning errors between the North component and the East

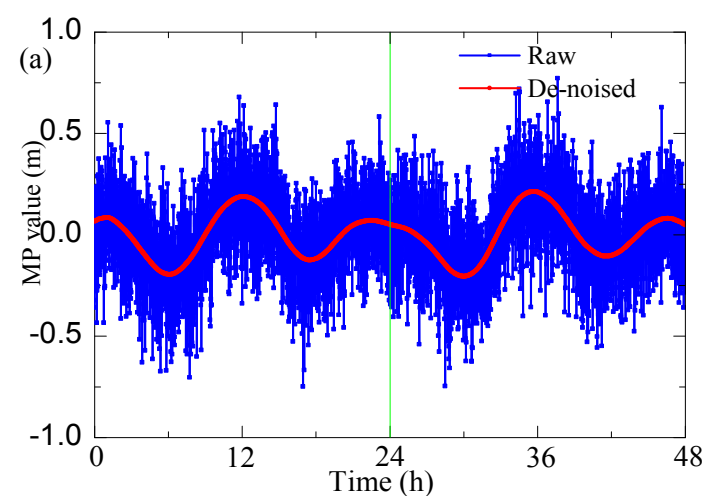

(a) Raw MP time series and de-noised time series

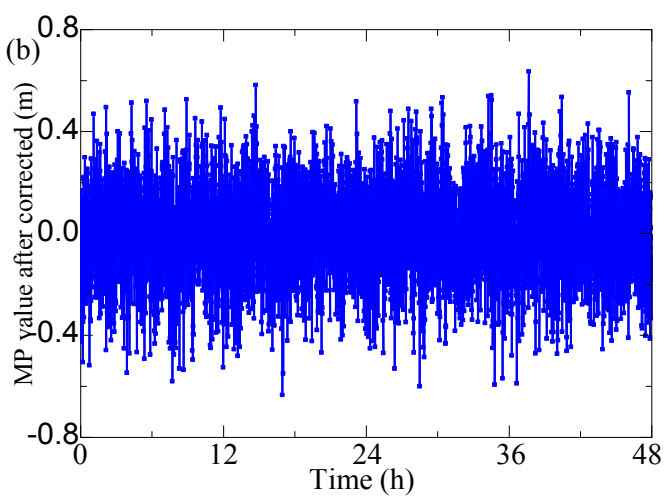

(b) Corrected MP time series

Fig. 6 MP series before and after code bias correction for C01 satellite. 


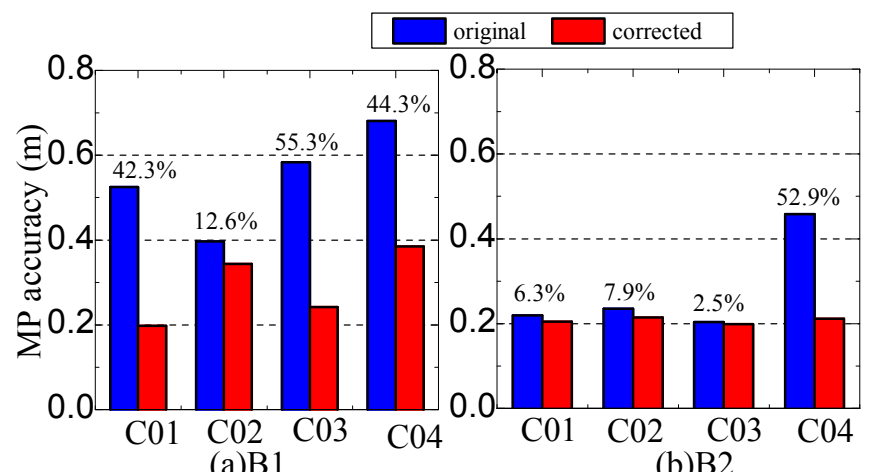

Fig. 7 Comparison of standard deviation for MP series before and after correction.
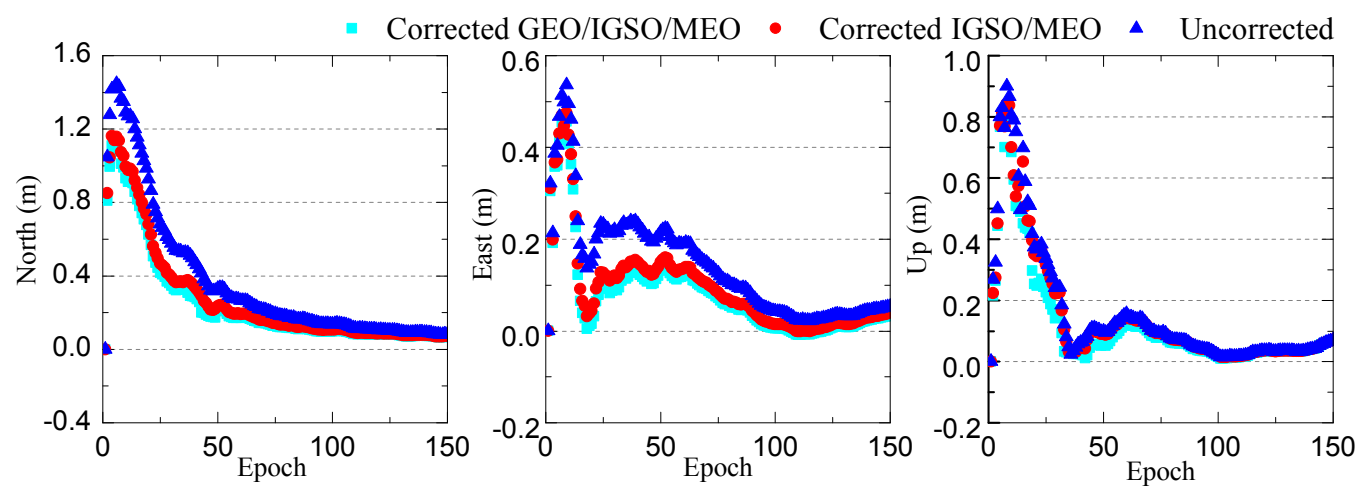

Fig. 8 Positioning errors for BDS PPP with three different strategies at the JFNG station.

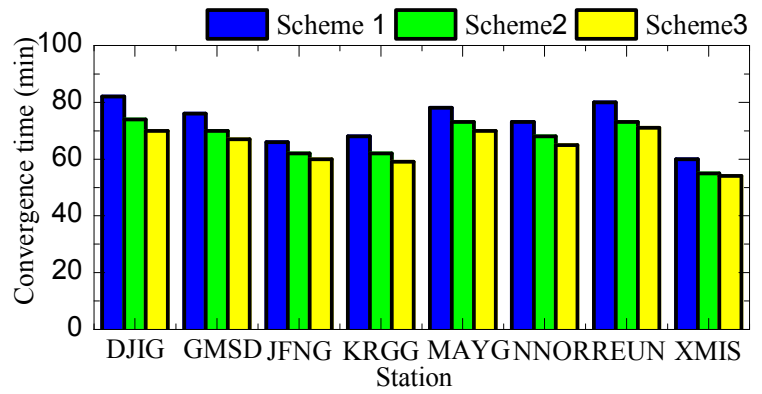

Fig. 9 Mean convergence time for BDS PPP with three different strategies at eight stations.

component are less than $10 \mathrm{~cm}$. Filtering converges at a certain epoch if positioning errors of the last 20 epochs remain within the limit. Figure 8 describes the positioning errors of BDS PPP with the above three strategies at the JFNG station on August 1, 2015.

As shown in Figure 8, the static PPP solution has been significantly improved by code bias correction in terms of its convergence time, with Scheme 3 being the best among these three strategies. The difference among them becomes very small when the convergence is completed. This is mainly because the PPP solution depends primarily on the carrier phase observation at high precision, and limited code bias correction can create improvement for the positioning accuracy in the PPP solution. The ambiguity is still not convergent in the initial phase of PPP, at which time the code bias plays a decisive role and its correction can effectively reduce the timespan needed for convergence. Figure 9 presents the mean convergence time for three strategies in static BDS PPP at eight stations. Compared with Scheme 1, mean convergence time is decreased by $7.9 \%$ with Scheme 2 , and further by $11.4 \%$, with Scheme 3 , proving that Scheme 3 (correction of GEO/IGSO/MEO code bias) is more effective at shortening the PPP convergence time. The root mean square (RMS) of the positioning deviation after static PPP convergence is calculated for the evaluation of positioning stability. As statistically listed in Table 2, the positioning accuracies of the PPP solution obtained from Scheme 3 are improved by $15.0 \%$ for the North component, $12.0 \%$ for the East component, and $5.0 \%$ for the Up

Table 2 Positioning errors for BDS PPP with three different strategies.

\begin{tabular}{lccc}
\hline $\begin{array}{l}\text { Positioning } \\
\text { errors }\end{array}$ & Scheme 1 & Scheme 2 & Scheme 3 \\
\hline $\mathrm{N}$ & 0.025 & 0.020 & 0.017 \\
$\mathrm{E}$ & 0.030 & 0.025 & 0.023 \\
$\mathrm{U}$ & 0.068 & 0.060 & 0.055 \\
\hline
\end{tabular}




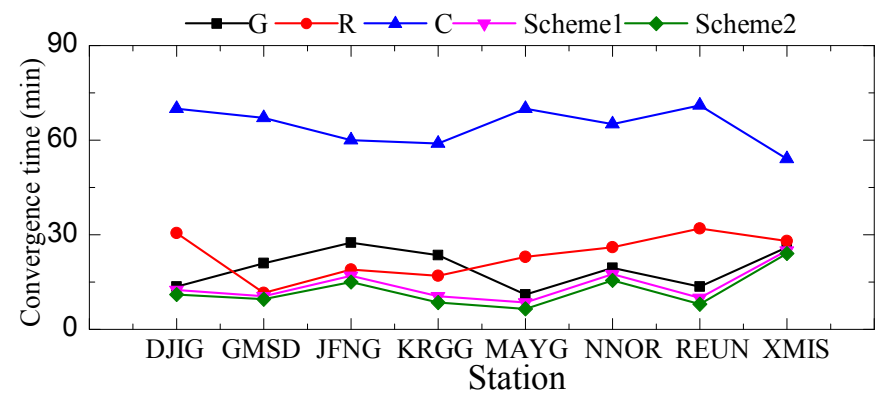

Fig. 10 Convergence times of different single systems and combined systems.

Table 3 The positioning errors of the multi-system PPP solution $(\mathrm{cm})$.

\begin{tabular}{ccccccc}
\hline Station & \multicolumn{3}{c}{ Scheme 1 } & \multicolumn{3}{c}{ Scheme 2 } \\
\cline { 2 - 7 } & $\mathrm{N}$ & $\mathrm{E}$ & $\mathrm{U}$ & $\mathrm{N}$ & $\mathrm{E}$ & $\mathrm{U}$ \\
\hline DJIG & 0.8 & 0.4 & 1.2 & 0.7 & 0.3 & 1.1 \\
GMSD & 0.4 & 0.7 & 1.4 & 0.4 & 0.8 & 1.3 \\
JFNG & -0.5 & 0.6 & 1.7 & -0.3 & 0.5 & 1.8 \\
KRGG & 0.4 & 0.9 & 0.8 & 0.3 & 0.7 & 0.3 \\
GMAY & 0.7 & 0.3 & 0.8 & 0.5 & 0.1 & 0.6 \\
NNOR & 0.5 & 0.3 & 0.7 & 0.4 & 0.1 & 0.7 \\
REUN & 0.3 & 0.7 & 0.5 & 0.1 & 0.5 & -0.4 \\
XMIS & 0.8 & 0.5 & 1.0 & 0.7 & 0.3 & 0.9 \\
\hline
\end{tabular}

Table 4 Positioning errors of the single-system PPP solution (cm).

\begin{tabular}{|c|c|c|c|c|c|c|c|c|c|}
\hline \multirow[t]{2}{*}{ Station } & \multicolumn{3}{|c|}{$\mathrm{G}$} & \multicolumn{3}{|c|}{$\mathrm{R}$} & \multicolumn{3}{|c|}{$\mathrm{C}$} \\
\hline & $\mathrm{N}$ & $\mathrm{E}$ & $\mathrm{U}$ & $\mathrm{N}$ & $\mathrm{E}$ & $\mathrm{U}$ & $\mathrm{N}$ & $\mathrm{E}$ & $\mathrm{U}$ \\
\hline DJIG & 0.8 & 0.6 & 1.9 & 0.5 & 0.8 & 1.3 & -0.4 & -0.8 & -3.6 \\
\hline GMSD & 0.5 & -0.2 & 0.7 & 0.3 & 0.2 & 1.4 & -2.9 & 2.3 & 5.6 \\
\hline JFNG & 0.1 & 0.9 & 2.1 & -0.5 & 0.7 & 2.9 & -3.1 & 2.9 & 5.2 \\
\hline KRGG & 0.2 & 0.4 & 0.5 & 0.2 & 0.7 & 0.9 & 1.2 & 4.0 & 4.7 \\
\hline GMAY & 0.7 & -0.2 & 0.6 & 0.5 & 0.2 & 0.9 & 2.4 & -3.2 & 5.4 \\
\hline NNOR & 0.2 & 0.1 & 0.5 & 0.6 & 0.4 & 0.6 & 3.0 & 2.3 & 5.0 \\
\hline REUN & 0.2 & -0.6 & -0.4 & -0.2 & 0.4 & -0.6 & -2.0 & 4.5 & 5.6 \\
\hline XMIS & 0.7 & 0.1 & 0.6 & 0.7 & 0.2 & 0.3 & -1.9 & -1.0 & 2.6 \\
\hline
\end{tabular}

directions compared with Scheme 2, and by $22.7 \%$, $21.4 \%$, and $12.3 \%$, respectively when compared with Scheme 1.

\section{STATIC PPP}

In order to analyze the effect of the BDS code bias on multi-GNSS PPP and the advantages of multiGNSS PPP, two schemes for the multi-GNSS PPP were used: Scheme 1, in which the BDS code bias is not corrected, and Scheme 2, in which the BDS code bias is corrected. The positioning performance of single and multi-GNSS PPP are analyzed from the aspects of positioning accuracy and convergence time. Figure 10 shows the convergence time of different single systems and combined systems. Table 3 shows the positioning errors for multi-system PPP with different schemes. Table 4 demonstrates the positioning errors for static PPP in a single-system using the 24-h data from August 6, 2015 (DOY218) at eight stations, respectively.

As shown in Figure 10, the multi-system PPP solution has been improved by the BDS code bias correction in terms of its convergence time. Table 3 shows that the positioning accuracy of the two schemes as being equal. This is mainly because the multi-GNSS PPP solution depends primarily on highprecision carrier phase observation.

According to data at the eight stations shown in Figure 10, GPS, GLONASS, and BDS need 19, 23, and $60 \mathrm{~min}$, respectively, to converge to positioning accuracy of centimeter level. GPS and GLONASS exhibit similar timespans for convergence while BDS needs more time. After the fusion of multiple systems, accelerated PPP convergence leads to a much reduced time of only $13 \mathrm{~min}$ for the combined GPS/GLONASS/BDS/Galileo system to converge. Table 4 suggests that GPS and GLONASS share the same positioning accuracy-better than $1 \mathrm{~cm}$ for the horizontal component and better than $3 \mathrm{~cm}$ for the vertical direction. However, BDS possesses slightly worse accuracy, with a horizontal precision better than $4 \mathrm{~cm}$ and vertical precision better than $6 \mathrm{~cm}$. This is mainly due to the fact that BDS is built and operated preliminarily with lower precision of BDS orbit- and clock-error products as well as a smaller number of satellites. The combined 


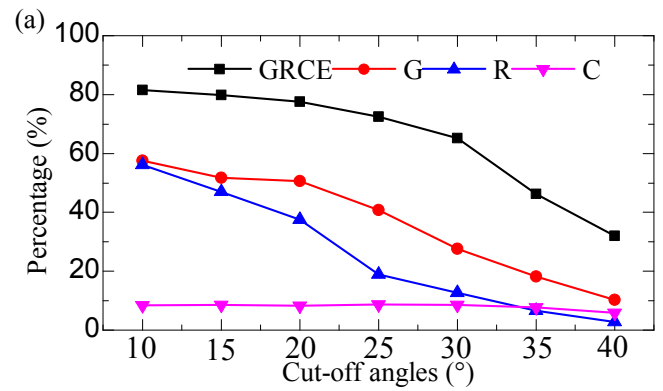

(a) Convergence percentage

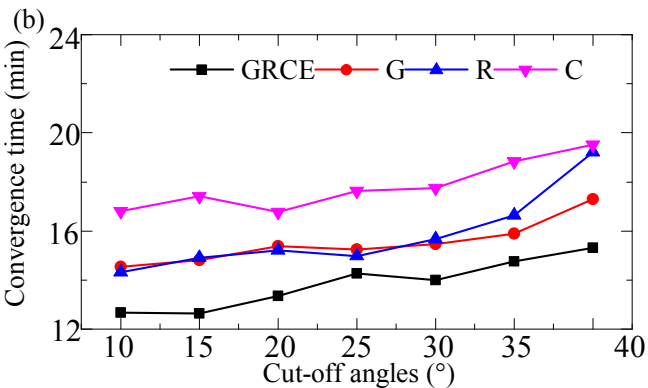

(b) Average convergence time

Fig. 11 Convergence percentage and average convergence time at different cut-off elevation angles.

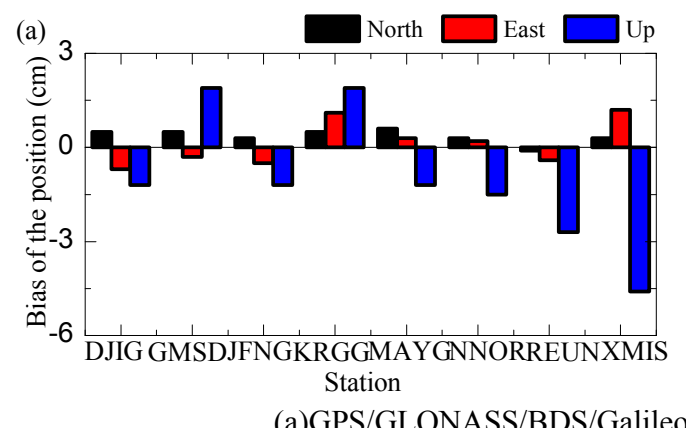

(a)GPS/GLONASS/BDS/Galileo

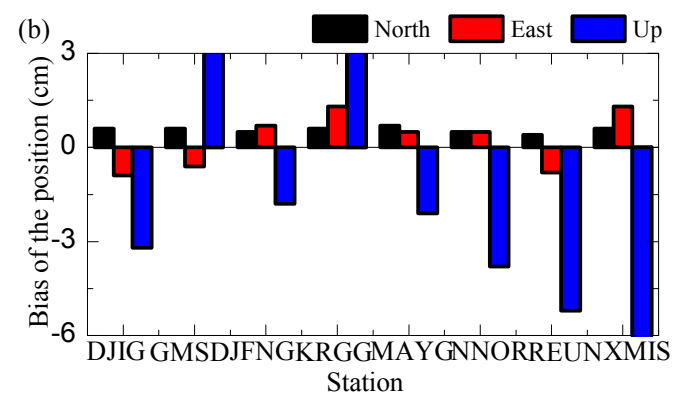

(b) GPS

Fig. 12 The positioning accuracy of the PPP solution at the cut-off elevation angle of $40^{\circ}$.

GPS/GLONASS/BDS/Galileo system can provide users with multiple usable satellites, a superior spatially geometric structure with high geometric accuracy for observation, and further improve the results of single GNSS positioning. The combined system ensures an average horizontal accuracy better than $1 \mathrm{~cm}$ and average vertical accuracy better than $2 \mathrm{~cm}$.

For a better demonstration of the advantages in multiple systems, 7-day consecutive observation data from eight stations have been intercepted to obtain 3360 sets of data with an observation time of $0.5 \mathrm{~h}$. The cut-off angles of satellite elevation cover the angle range between $10^{\circ}$ and $40^{\circ}$ with an interval of $5^{\circ}$ for simulation of urban canyons or obstructed areas, and the rate for convergence to the centimeter level under different scenarios and modes has been analyzed, along with the positioning accuracy. Figure 11 describes the variation of convergence percentage and average convergence time with cutoff angles. Figure 12 presents the positioning accuracy of the PPP solution in GPS and GPS/GLONASS/BDS/Galileo models when the cutoff angle for satellite elevation is $40^{\circ}$.

As shown in Figure 11, the convergence percentage decreases with increasing cut-off angle, and the GPS/GLONASS/BDS/Galileo model with combined system exhibits better positioning performance than other single-system models do when they are at the same elevation. For instance, the GPS/GLONASS/BDS/Galileo model achieves $77.52 \%$ of convergence within an average timespan of $13.226 \mathrm{~min}$ at a cut-off elevation angle of $20^{\circ}$, which is superior to single-system models. Figure 12 further proves that positioning results in the multi-system GPS/GLONASS/BDS/Galileo model are better than those in the single GPS model at a cut-off angle of $40^{\circ}$, especially the significant improvement of elevation direction. At higher cut-off elevation angle, the number of visible satellites in the single GPS system decreases rapidly, while that in the multisystem combination remains more than 10 , which is why fusion positioning in a multi-system model possesses better accuracy and stability than the singlesystem model.

\section{CONCLUSIONS}

In this research, an EMD-based correction approach is proposed for the multi-GNSS PPP observation model, which focuses on code bias correction of the GEO satellites in BDS. For model verification, PPP solutions in both BDS and multiGNSS models have been computed with observation data at eight MGEX stations for seven consecutive days, and the conclusions obtained are listed below.

Along with the weakened code bias of BDS GEO, the standard deviation of MP series on frequencies $\mathrm{B} 1$ and $\mathrm{B} 2$ decreased by $38.63 \%$ and $17.4 \%$, respectively. In the meantime, the timespan required by PPP convergence in BDS exhibited an $11.4 \%$ decrease after GEO/IGSO/MEO code bias correction. At present, the PPP solution in BDS possesses inferior accuracy of positioning, as well as a longer timespan for convergence, compared with both GPS-only and GLONASS-only models due to the limited accuracy of products in the BDS system. 
Conversely, the PPP solution in the combined GPS/GLONASS/BDS/Galileo model shows a positioning accuracy equivalent to those of the GPS and GLONASS models, and its convergence time has been improved. Since there are less visible satellites at high cut-off angles of elevation, positioning can be affected to a large extent. At an elevation of $20^{\circ}$, however, $77.52 \%$ for convergence is still achieved by the PPP solution in the GPS/GLONASS/BDS/Galileo model within 13.26 min of mean convergence time. Therefore, the multi-GNSS has contributed greatly to the acquisition of highly accurate positioning.

\section{ACKNOWLEDGMENTS:}

We thank the anonymous reviewers for their helpful suggestions. This work is supported by the National Natural Science Foundation of China (Nos. 41604018 and 41704008), the Fundamental Research Funds for the Central Universities (No. 2019B17514) and the Program for Excellent Talents in University of Anhui Province of China (No. gxyq2017008). We also thank the IGS communities for the provision of GNSS data and products.

\section{REFERENCES}

Abd Rabbou, M. and El-Rabbany, A.: 2016, Performance analysis of precise point positioning using multiconstellation GNSS: GPS, GLONASS, Galileo and BeiDou. Surv. Rev., 49, 352, 39-50. DOI: 10.1080/00396265.2015.1108068

Cai, C., Gao, Y., Pan, L. and Zhu, J.: 2015, Precise point positioning with quad-constellations: GPS, BeiDou, GLONASS and Galileo. Adv. Space Res., 56, 1, 133143. DOI: $10.1016 /$ j.asr.2015.04.001

Cai, C. and Gao, Y.: 2012, Modeling and assessment of combined GPS/GLONASS precise point positioning. GPS Solut., 17, 2, 223-236. DOI: $10.1007 / \mathrm{s} 10291-012-0273-9$

Guo, F., Li, X., Zhang, X. and Wang, J.: 2016, The contribution of Multi-GNSS Experiment (MGEX) to precise point positioning. Adv. Space Res., 59, 11, 2714-2725. DOI:10.1016/j.asr.2016.05.018

Jokinen, A., Feng, S., Schuster, W., Ochieng, W., Hide, C., Moore, T. and Hill, C.: 2013, GLONASS aided GPS ambiguity fixed precise point positioning. J. Navigation, 66, 3, 399-416.

DOI: $10.1017 / \mathrm{s} 0373463313000052$

Ge, M., Gendt, G., Rothacher, M., Shi, C. and Liu, J.: 2007, Resolution of GPS carrier-phase ambiguities in Precise Point Positioning (PPP) with daily observations. J. Geodesy, 82, 7, 389-399. DOI: $10.1007 / \mathrm{s} 00190-007-0187-4$

Hauschild, A., Montenbruck, O., Sleewaegen, J., Huisman, L. and Teunissen, P.J.G.: 2012, Characterization of Compass M-1 signals. GPS Solut., 16, 1, 117-126. DOI: 10.1007/s10291-011-0210-3

Li, P. and Zhang, X.: 2013, Integrating GPS and GLONASS to accelerate convergence and initialization times of precise point positioning. GPS Solutions, 18, 3, 461471. DOI: $10.1007 / \mathrm{s} 10291-013-0345-5$

Li, X., Ge, M., Dai, X., Ren, X., Fritsche, M., Wickert, J. and Schuh, H.: 2015, Accuracy and reliability of
multi-GNSS real-time precise positioning: GPS, GLONASS, BeiDou, and Galileo. J. Geodesy, 89, 6, 607-635. DOI: 10.1007/s00190-015-0802-8

Liu, T., Yuan, Y., Zhang, B., Wang, N., Tan, B. and Chen, Y.: 2017, Multi-GNSS precise point positioning (MGPPP) using raw observations. J. Geodesy, 91, 3, 253-268. DOI: 10.1007/s00190-016-0960-3

Liu, Z.Q., Wang, J.X. and Duan, B.B.: 2015, Estimation of GLONASS code inter-frequency biases with multiple parameters based on a single station and its impact on combined precise point positioning. Acta Geod. Cartographica Sinica., 44, 2, 150-159. DOI:10.11947/j.AGCS.2015.20130800

Lou, Y., Gong, X., Gu, S., Zheng, F. and Feng, Y.: 2016, Assessment of code bias variations of BDS triplefrequency signals and their impacts on ambiguity resolution for long baselines. GPS Solut., 21, 1, 177186. DOI: 10.1007/s10291-016-0514-4

Martín, A., Anquela, A. B., Capilla, R. and Berné, J. L.: 2011, PPP technique analysis based on time convergence, repeatability, IGS products, different software processing, and GPS+GLONASS constellation. J. Surv. Eng., 137, 3, 99-108. DOI: $10.1061 /($ asce)su. 1943-5428.0000047

Montenbruck, O., Hauschild, A., Steigenberger, P., Hugentobler, U., Teunissen, P., and Nakamura, S.: 2012, Initial assessment of the COMPASS/BeiDou-2 regional navigation satellite system. GPS Solut., 17, 2, 211-222. DOI: 10.1007/s10291-012-0272-x

Ren, X.D., Zhang, K.K., Li, X.X. and Zhang, X.H.: 2015, Precise point positioning with multi-constellation satellite systems: BDS, Galileo, GLONASS, GPS. Acta Geod. Cartographica Sinica, 44, 12, 1307-1339. DOI:10.11947/j.AGCS.2015.20140568

Wang, G., de Jong, K., Zhao, Q., Hu, Z. and Guo, J.: 2014, Multipath analysis of code measurements for BeiDou geostationary satellites. GPS Solut., 19, 1, 129-139. DOI: $10.1007 / \mathrm{s} 10291-014-0374-8$

Wanninger, L. and Beer, S.: 2014, BeiDou satellite-induced code pseudorange variations: diagnosis and therapy. GPS Solut., 19,4, 639-648. DOI: $10.1007 / \mathrm{s} 10291-014-0423-3$

Wu, X., Zhou, J., Wang, G., Hu, X. and Cao, Y.: 2012, Multipath error detection and correction for GEO/IGSO satellites. Sci. China Phys. Mech. Astron., 55, 7, 1297-1306. DOI: 10.1007/s11433-012-4741-6

Zhao, Q., Guo, J., Li, M., Qu, L., Hu, Z., Shi, C. and Liu, J.: 2013, Initial results of precise orbit and clock determination for COMPASS navigation satellite system. J. Geodesy, 87, 5, 475-486. DOI: $10.1007 / \mathrm{s} 00190-013-0622-7$

Zhao, X., Wang, S., Liu, C., Ou, J. and Yu, X.: 2016, Assessing the performance of multi-GNSS precise point positioning in Asia-Pacific region. Surv. Rev., 49, 354, 186-196. DOI:10.1080/00396265.2016.1151576

Zhang, X. H., Zuo, X., Li, P. and Pan, Y.M.: 2015, Convergence time and positioning accuracy comparison between BDS and GPS precise point positioning. Acta Geod. Cartographica Sinica, 44, 3, 250-256. DOI:10.11947/j.AGCS.2015.20130771

Zhu, Y.X., Feng, L.P., Jia, X.L., Zhang, Q.H. and Ruan, R.G.: 2015,The PPP precision analysis based on BDS regional navigation system. Acta Geod. Cartographica Sinica, 44, 4, 377-383. DOI:10.11947/j.AGCS.2015.20140082 